\title{
USING OF SELF-TUNING CONTROLLERS SIMULINK LIBRARY FOR REAL-TIME CONTROL OF NONLINEAR SERVO SYSTEM
}

\author{
Petr Chalupa, Vladimír Bobál, Jakub Novák and Petr Dostál \\ Department of Process Control, Faculty of Applied Informatics \\ Tomas Bata University in Zlín \\ Nad Stráněmi 4511, 76005 Zlín, Czech Republic \\ E-mail:bobal@fai.utb.cz
}

\section{KEYWORDS}

Self-tuning control, ARX model, recursive least squares, LQ control, polynomial approach, MATLABSimulink, servo motor, real-time verification

\begin{abstract}
The combination of the automatic control theory courses, simulation verification and practical implementation of the designed controller algorithms in real-time conditions is very important for training of the control engineers. This contribution present structure and usage of Self-tuning Controllers Simulink Library (STCSL) for real time control. The STCSL was created for design, simulation verification and especially realtime implementation of single input - single output (SISO) digital self-tuning controllers. The proposed adaptive controllers, which are included in the library, can be divided into three groups (PID controllers, controllers based on the polynomial approach and the controllers derived on the other approaches (minimum variance etc.). This Library is very successfully used in Adaptive Control Course in education practice for design and verification of self-tuning control systems in simulation and real-time conditions. It is suitable also for design and verification of industrial digital controllers. The highly nonlinear laboratory model, the DR300 Speed Control with Variable Load, has been chosen as example for real-time control. The STCSL is available free of charge at the Tomas Bata University internet site - http://www.utb.cz/stctool/.
\end{abstract}

\section{INTRODUCTION}

One approach to adaptive control is based on recursive estimation of unknown system characteristics, gradually specifying them, and then monitoring possible changes. Using this knowledge, appropriate methods can be employed to design the optimal controller. This kind of controller, which identifies unknown processes and then synthesizes control (adaptive control with recursive identification), is referred to in the literature as a selftuning controller (STC). Self-tuning controllers use the combination of the recursive process identification based on a selected model process and a controller synthesis based on knowledge of parameter estimates of controlled process.
In some STCs, the identification process does not serve to determine the estimates of the process model parameters $\hat{\Theta}(k)$; rather, appropriate reparametrisation of the control loop can be used recursively to estimate the controller parameters directly. Therefore, it is necessary to find the relationship between the process input and output and define it straight from the controller parameters without recalculating them using the estimates of the process model parameters. These controllers are referred to as being implicit, whereas controllers using a synthesis from estimates of the process model parameters are called explicit (with direct identification).

Presently, most of the explicit STCs are based on the Certainty Equivalence (CE) Principle. The basic principle of $\mathrm{CE}$ is that the model uncertainty is not considered. The parameter estimates of the process model, which are obtained by recursive identification, are used for the controller design. It is assumed that values of these estimates correspond to their actual values.

The aim of this contribution is not only to inform potential users about Self-tuning Controllers Simulink Library - STCSL (see Bobál and Chalupa, 2002) and to provide help for usage of the library in the simulation verification (see Bobál et al. 2008) but also to demonstrate the practical approach to a design of a control of the laboratory model in real-time conditions.

\section{RECURSIVE IDENTIFICATION}

The regression (ARX) model of the following form

$$
y(k)=\boldsymbol{\Theta}^{T}(k) \boldsymbol{\Phi}(k-1)+n(k)
$$

is used in the identification part of the designed controller algorithms, where

$$
\boldsymbol{\Theta}^{T}(k)=\left[a_{1}, a_{2}, \ldots, a_{n a}, b_{1}, b_{2}, \ldots, b_{n b}\right]
$$

is the vector of the parameters and

$$
\begin{aligned}
\boldsymbol{\Phi}^{T}(k-1)= & {[-y(k-1),-y(k-2), \ldots,-y(k-n a),} \\
& u(k-1), u(k-2), \ldots, u(k-n b)]
\end{aligned}
$$


is the regression vector $(y(k)$ is the process output variable, $u(k)$ is the controller output variable). The non-measurable random component $n(k)$ is assumed to have zero mean value $E[n(k)]=0$ and constant covariance (dispersion) $R=E\left[n^{2}(k)\right]$.

The recursive least squares method for calculating of parameter estimates $\hat{\boldsymbol{\Theta}}(k)$ is utilized. Using the pure least squares method, the influence of all pairs of identified system inputs and outputs to the parameters estimates is the same. This property can be inconvenient for example when identifying a system with timevarying parameters. In this case, it is better to use least squares method with exponential forgetting where the influence of latter data to the calculation of the parameter estimates is greater then the influence of older data.

The exponential forgetting method can be further improved by adaptive directional forgetting (Kulhavý, 1987) which changes forgetting coefficient with respect to changes of input and output signal.

\section{CONTROLLER ALGORITHMS}

The proposed self-tuning controllers which are included in the library are divided into three groups:

- classical digital PI and PID controllers whose tuning is based on Ziegler-Nichols method, pole assignment method and its modifications,

- controllers based on polynomial approach (deadbeat strong and weak version, pole assignment, controllers based on minimization of quadratic criterion),

- controllers based on other approaches (minimum variance controllers, Dahlin's controller, BányászKeviczky's controller etc.).

\section{SELF-TUNING CONTROLLERS SIMULINK LIBRARY}

The Simulink is nowadays a word-wide standard in simulation, testing, and verification of behaviour of various dynamic systems. Simulink is a part of MATLAB system and supports linear or nonlinear systems modelled in continuous time, sampled time or a hybrid of the two. Systems can also be multirate, i.e. have different parts that are sampled or updated at different rates.

Based on monograph Bobál, et al. (2005) a library of self-tuning controllers in MATLAB/Simulink environment was created. The purpose was to create a framework suitable for creating and testing of selftuning controllers. The library is available free of charge at internet site of Tomas Bata University in Zlín - www.utb.cz/stctool/ (see Bobál and Chalupa, 2002). The library was created using MATLAB version 6.5 (Release 13) and is compatible with all newer
MATLAB versions. Controllers are implemented in the library as standalone Simulink blocks, which allows an easy incorporation into existing simulation schemes and an easy creation of new simulation circuits. Only standard techniques of Simulink environment were used when creating the controller blocks and thus just basic knowledge of this environment is required to start working with the library. Controllers can be implemented into simulation schemes just by the copy or drag \& drop operation and their parameters are set using dialog windows. Another advantage of the used approach is a relatively easy implementation of userdefined controllers by modifying some suitable controller from the library.

Nowadays the library contains over 30 simple single input single output discrete self-tuning controllers, which use discrete ARX models of second and third order for the on-line system identification.

\section{DESCRIPTION OF LABORATORY MODEL AMIRA DR300}

Some of self-tuning algorithms were tested using a realtime laboratory model DR300 (Speed Control with Variable Load) by the Amira Company, Duisburg, Germany (see Fig. 1).

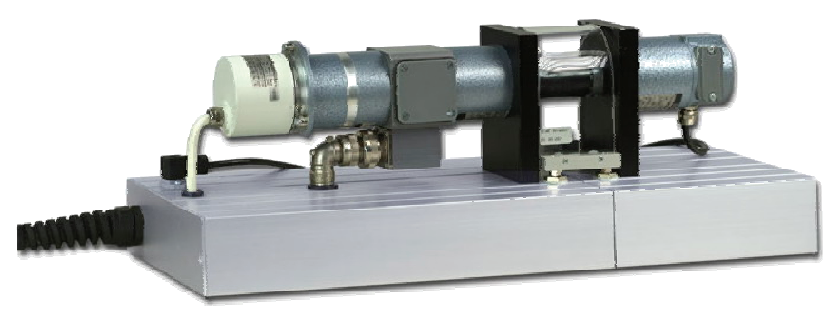

Figure 1: Laboratory Model Amira DR300

A simplified scheme of the DR300 system is shown in Fig. 2.

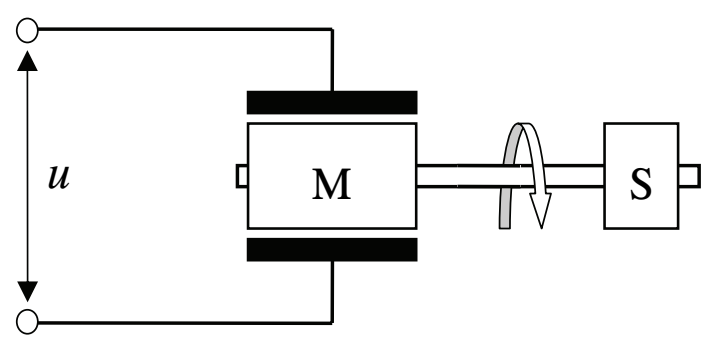

Figure: 2: Simplified Scheme of Amira DR300 Laboratory servo System ( $u$ :Motor Control Voltage; M: DC Motor; S: Motor Shaft Rotation Sensor)

The rotation speed of the motor $M$ is driven by voltage $u$. The motor shaft rotations per minute (rpm) are measured by sensor S. The aim of the control process is to control the rotation speed of the shaft by the control voltage $u$. 


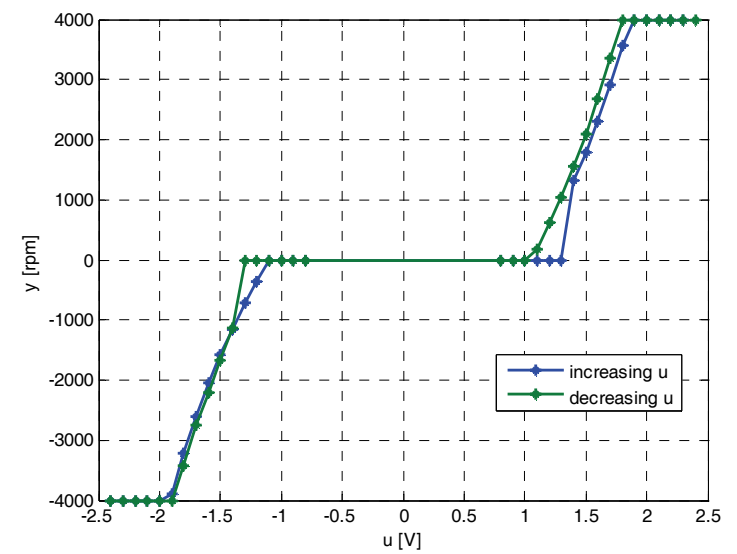

Figure 3: Static Characteristics of DR300 Servomotor

From the control point of view, the Amira DR300 is a nonlinear system. Some characteristics of the nonlinearity can be observed from the static characteristics shown in Fig. 3.

\section{EXPERIMENTAL RESULTS}

Many different setting of the self-tuning controller from the STCSL (Bobál, Chalupa 2002) were applied to the Amira DR300 system and some results are presented in this chapter.

Interconnection of a MATLAB/Simulink and the Amira DR300 system was realized using Real-Time Toolbox (RTT) and the MF614 data acquisition card (both by Humusoft). The Real-Time Toolbox allows direct connection of the Simulink and a real-time controlled system without any restrictions concerning the programming language that is used for user-defined Simulink functions. Contrary to Real-Time Workshop, the RTT can use whole set of MATLAB functions and toolboxes. Main drawback of usage RTT consists in the fact that the toolbox cannot produce source code for stand-alone controlled systems.

The LQ based controller $p p 2 l q$ was used as a base for comparison with other controllers and setting. Computation of the control sequence of the pp2lq controller is based on minimization of a quadratic criterion in the following form (Bobál et al. 2005). The coefficients of the characteristic polynomial $D\left(z^{-1}\right)$ of the closed loop are computed to minimize the LQ criterion (4):

$$
\begin{aligned}
& J=\sum_{k=0}^{\infty}\left\{[w(k)-y(k)]^{2}+\lambda[u(k)]^{2}\right\} \\
& D\left(z^{-1}\right)=1+d_{1} z^{-1}+d_{2} z^{-2} \\
& d_{1}=\frac{m_{1}}{\delta+m_{2}} ; \quad d_{2}=\frac{m_{2}}{\delta}
\end{aligned}
$$

The parameters $m_{1}, m_{2}$ and $\delta$ are computed as follows:

$$
\begin{aligned}
& \delta=\frac{\gamma+\sqrt{\lambda^{2}-4 m_{2}^{2}}}{2} ; \gamma=\frac{m_{0}}{2}-m_{2}+\sqrt{\left(\frac{m_{0}}{2}+m_{2}\right)^{2}-m_{1}^{2}} \\
& m_{0}=\lambda\left(1+a_{1}^{2}+a_{2}^{2}\right)+b_{1}^{2}+b_{2}^{2} ; m_{1}=\lambda\left(a_{1}+a_{1} a_{2}\right)+b_{1} b_{2} \\
& m_{2}=\lambda a_{2}
\end{aligned}
$$

where $\lambda$ is a coefficient representing weight of control signal increments in the LQ criterion.

The LQ controller of 2DOF (two degrees of freedom) structure was applied in the form

$$
\begin{aligned}
u(k) & =r_{0} w(k)-q_{0} y(k)-q_{1} y(k-1)-q_{2} y(k-2)+ \\
& +\left(1-p_{1}\right) u(k-1)+p_{1} u(k-2)
\end{aligned}
$$

The expressions for computation of individual controller parameters are introduced in Bobál, et al. (2005).

The sample time $T_{0}=0.05 \mathrm{~s}$, penalization of control sequence $\lambda=5$ (denoted as "Penalization of controller output - fi" in the STCSL) and on-line identification with the adaptive directional forgetting was used as a nominal case. Control courses obtained by these settings are presented in Fig. 4.
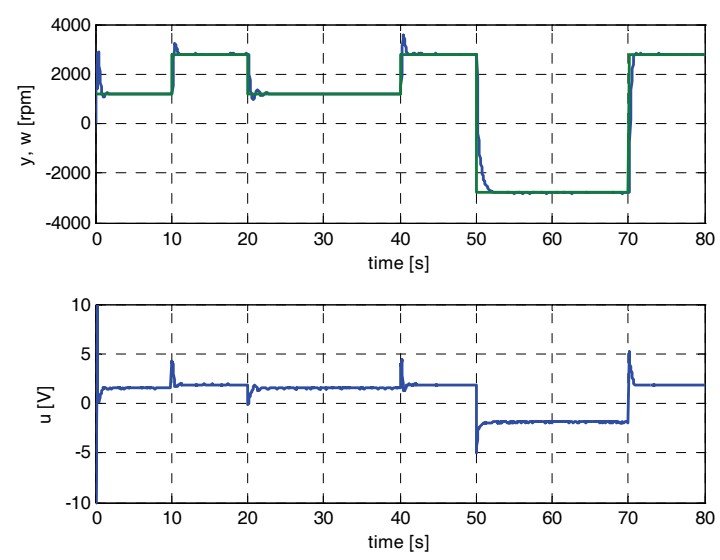

Figure 4: Control Courses of the Nominal Settings of pp $2 l q$ Controller $\left(T_{0}=0.05, a d f, \lambda=5\right)$

While courses presented in this figure are in physical units, the values of the controller input and output were normalized. The normalized controller inputs in the range $\langle-1,1\rangle$ represented rotation speed of the shaft in the range $\langle-4000 \mathrm{rpm}, 4000 \mathrm{rpm}\rangle$. The normalized control signal in the range $\langle-1,1\rangle$ represented control voltage in the range $\langle-10 \mathrm{~V}, 10 \mathrm{~V}\rangle$. It should be also noted that no a priori information about the controlled system was used in all control courses presented in this paper and therefore oscillations occur at the beginning of control process where the model does not represent the controlled system well. Performance of individual controllers was tested using summing criteria based on squares and on absolute values of both control error sequence and control sequence differences. 


$$
\begin{aligned}
& S_{e 2}=\frac{1}{N_{2}-N_{1}+1} \sum_{k=N_{1}}^{N_{2}}[e(k)]^{2} \\
& S_{d u 2}=\frac{1}{N_{2}-N_{1}} \sum_{k=N_{1}}^{N_{2}-1}[u(k+1)-u(k)]^{2} \\
& S_{e a}=\frac{1}{N_{2}-N_{1}+1} \sum_{k=N_{1}}^{N_{2}}|e(k)| \\
& S_{d u a}=\frac{1}{N_{2}-N_{1}} \sum_{k=N_{1}}^{N_{2}-1}|u(k+1)-u(k)|
\end{aligned}
$$

The values of $N_{1}$ and $N_{2}$ were set to cover whole control process except first $5 \mathrm{~s}$ - i.e. the time range for computation of summing criteria was $\langle 5 \mathrm{~s}, 80 \mathrm{~s}\rangle$.

\section{Influence of penalisation}

The first parameter, which was studied, was penalisation weight $\lambda$. Resulting criteria values are presented in Table 1 . The sample period $T_{0}=0.05 \mathrm{~s}$ was used and default values of all other parameters were applied.

Table 1: Influence of the Penalisation

\begin{tabular}{|c|r|r|c|c|}
\hline$\lambda$ & $S_{e 2}\left[10^{3}\right]$ & $S_{\text {du2 } 2}[1]$ & $S_{e a}[1]$ & $S_{\text {dua }}[1]$ \\
\hline $10^{-6}$ & 124.13 & 0.6779 & 41.5446 & 0.2329 \\
\hline 0.002 & 124.12 & 0.6687 & 41.8897 & 0.2387 \\
\hline 0.01 & 124.22 & 0.6658 & 41.6476 & 0.2268 \\
\hline 0.04 & 124.51 & 0.5445 & 40.5928 & 0.1723 \\
\hline 0.2 & 125.97 & 0.3525 & 42.4119 & 0.1328 \\
\hline 1 & 135.04 & 0.2664 & 46.8386 & 0.1001 \\
\hline 2 & 153.18 & 0.1576 & 57.9602 & 0.0682 \\
\hline 5 & 187.05 & 0.0815 & 76.1243 & 0.0518 \\
\hline 20 & 246.90 & 0.0415 & 103.6711 & 0.0387 \\
\hline 100 & 356.24 & 0.0418 & 141.6785 & 0.0545 \\
\hline
\end{tabular}

To simplify comparison of individual settings, criterion values were normalised and values related to quadratic criteria were summed as well as values related to absolute values

$$
\begin{aligned}
& S_{2}(i)=\frac{1}{2}\left[\frac{S_{e 2}(i)}{S_{e 2}(\text { nom })}+\frac{S_{d u 2}(i)}{S_{d u 2}(\text { nom })}\right] \\
& S_{a}(i)=\frac{1}{2}\left[\frac{S_{e a}(i)}{S_{e a}(\text { nom })}+\frac{S_{d u a}(i)}{S_{d u a}(\text { nom })}\right]
\end{aligned}
$$

The index nom in equation (8) represents the values achieved by the nominal controller - i.e. pp $2 l q$ controller with sample time $T_{0}=0.05$, penalisation $\lambda=5$, on-line identification with adaptive directional forgetting and not using a priori information. Values of the $S_{2}$ and $S_{a}$ based on Table 1 are summed up in Table 2 .
Table 2: Normalized Criteria for Different Penalisations

\begin{tabular}{|c|c|c|}
\hline$\lambda$ & $\mathrm{S}_{2}[1]$ & $\mathrm{S}_{\mathrm{a}}[1]$ \\
\hline $10^{-6}$ & 4.4927 & 2.5217 \\
\hline 0.002 & 4.4367 & 2.5798 \\
\hline 0.01 & 4.4188 & 2.4632 \\
\hline 0.04 & 3.6752 & 1.9306 \\
\hline 0.2 & 2.5007 & 1.5610 \\
\hline 1 & 1.9960 & 1.2738 \\
\hline 2 & 1.3769 & 1.0393 \\
\hline 5 & 1.0000 & 1.0000 \\
\hline 20 & 0.9144 & 1.0546 \\
\hline 100 & 1.2086 & 1.4565 \\
\hline
\end{tabular}

It is possible to present the values from Table 2 in a graphical form as shown in Fig. 5.

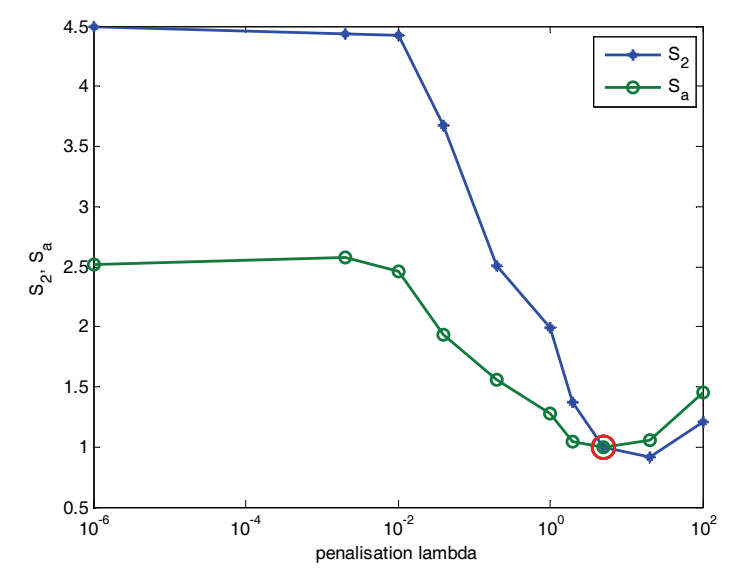

Figure 5: $S_{2}$ and $S_{a}$ Criteria for $p p 2 l q$ with Different $\lambda$

The nominal settings of the $p p 2 l q$ controller are marked by circle. The optimal value of the $\lambda$ parameter were $\lambda=20\left(S_{2}\right.$ criterion $)$ and $\lambda=5\left(S_{a}\right.$ criterion $)$.

\section{Influence of sample time}

The same technique as described in the previous subchapter was used to study the influence of the sample time. The penalization $\lambda=5$ was used and all the other parameters remained on their nominal values. Resulting criteria values are summarized in Table 3.

Table 3: Influence of Sample Time

\begin{tabular}{|c|c|c|}
\hline$T_{0}$ & $S_{2}[1]$ & $S_{a}[1]$ \\
\hline 0.005 & 1.1218 & 2.2919 \\
\hline 0.01 & 0.6886 & 1.6190 \\
\hline 0.025 & 0.6739 & 1.0115 \\
\hline 0.05 & 1.0000 & 1.0000 \\
\hline 0.1 & 1.6034 & 1.3025 \\
\hline 0.2 & 3.1799 & 2.9034 \\
\hline
\end{tabular}


The values from the Table 3 are presented in Fig. 6 . Optimal value of sample time $T_{0}=0.025 \mathrm{~s}$ was obtained according to $S_{2}$ criterion and $T_{0}=0.05 \mathrm{~s}$ according to $S_{a}$ criterion.

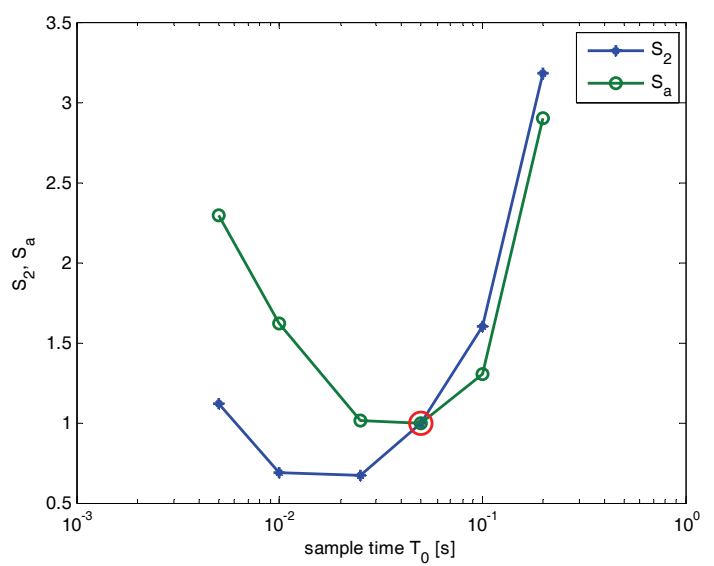

Figure 6: $S_{2}$ and $S_{a}$ Criteria for $p p 2 l q$ with Different $T_{0}$

The control courses of $T_{0}=0.025 \mathrm{~s}$ are presented in Fig. 7.
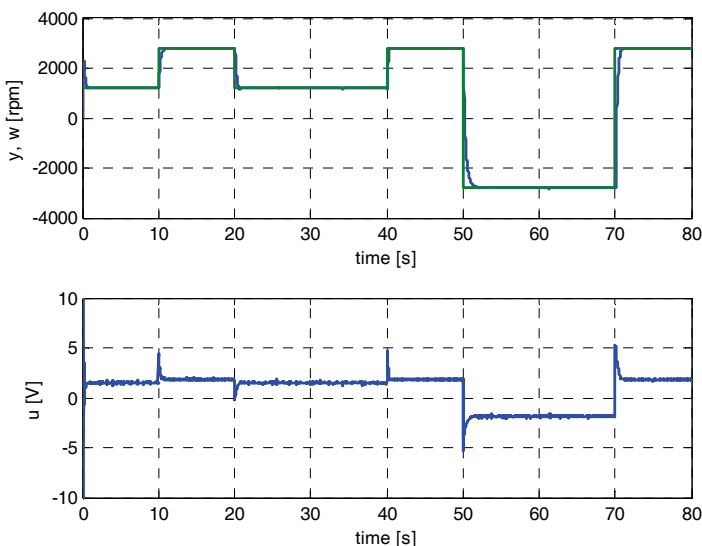

Figure 7: Control Courses of $p p 2 l q, T_{0}=0.025 \mathrm{~s}$

It can be seen that tracking performance of the control system has increased comparing to sample time $T_{0}=0.05$ (see Fig. 4). On the other hand, greater changes of control signal occurred at the moments of set-point changes.

\section{Influence of forgetting}

The last series of experiments performed entirely using $p p 2 l q$ controller was focused on the influence of forgetting to the control process. The problem was studied using exponential forgetting where the forgetting coefficient $\varphi$ was being changed in the range $\langle 0.6,1\rangle$. Resulting criteria values are summarized in Table 4, nominal case using adaptive directional forgetting $(a d f)$ is also included.
Table 4: Influence of Forgetting Coefficient

\begin{tabular}{|c|l|c|}
\hline$\varphi$ & $S_{2}[1]$ & $S_{a}[1]$ \\
\hline 0.6 & 3.6062 & 2.3817 \\
\hline 0.7 & 1.5174 & 1.2230 \\
\hline 0.8 & 0.9720 & 0.8354 \\
\hline 0.9 & 0.9654 & 0.8679 \\
\hline 0.95 & 0.9795 & 0.9880 \\
\hline 0.98 & 1.1373 & 1.2870 \\
\hline 1 & 1.5944 & 2.8767 \\
\hline$a d f$ & 1.0000 & 1.0000 \\
\hline
\end{tabular}

The courses of criteria values from the Table 4 are presented in Fig. 8.

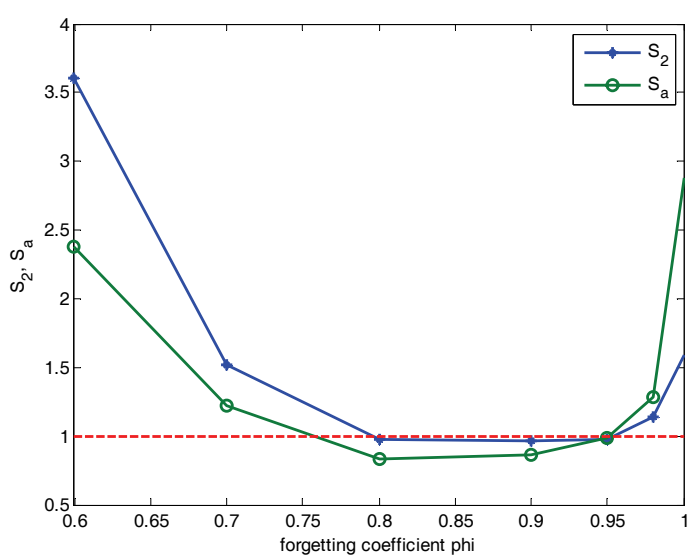

Figure 8: $S_{2}$ and $S_{a}$ Criteria for $p p 2 l q$ with Different $\varphi$

The values corresponding to nominal settings with adaptive directional forgetting are represented by the dashed line. Optimal value of forgetting coefficient according to $\mathrm{S} 2$ criterion is $\varphi=0.9$. According to $\mathrm{Sa}$ criterion, the optimal value $\varphi=0.8$ was obtained.

The value of $\varphi=1$ represents the situation where the weights of all data pairs are the same and therefore exponential forgetting is transformed to pure recursive least squares method (RLSM). On-line identification does not reach satisfactory performance and tracking of the reference signal was not achieved as can be from the control courses presented in Fig. 9.
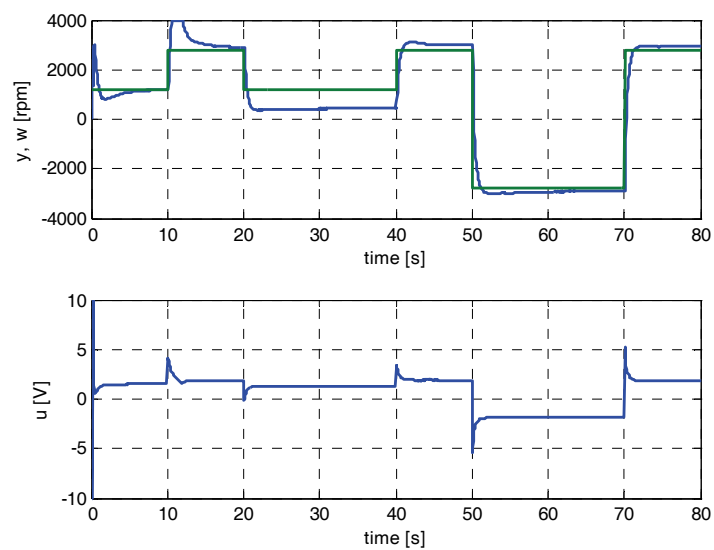

Figure 9: Control Courses of $p p 2 l q$ Using Pure RLSM 


\section{Influence of control algorithm}

The control algorithm plays the most important role when comparing different controllers and their settings. The algorithm is used to compute controller parameters from the current estimates of the parameters of the controlled system model and subsequently compute control sequence. This subchapter contains comparison of several control algorithms from the STCSL. The nominal sample time of $T_{0}=0.05 \mathrm{~s}$ and adaptive directional forgetting were used in all cases. The other parameters as well as the values of $S_{2}$ and $S_{a}$ are shown in Table 5.

Table 5: Performance of Different Control Algorithms

\begin{tabular}{|l|l|l|l|}
\hline algorithm & $S_{2}[1]$ & $S_{a}[1]$ & parameters \\
\hline$d b 2 s$ & 4.5380 & 2.4122 & \\
\hline mv2 & 1.8083 & 4.3959 & $q=10$ \\
\hline pp2a_1 & 1.4491 & 1.2384 & $\xi=1, \omega=2$ \\
\hline pp2b_1 & 1.6135 & 1.8294 & $\xi=1, \omega=2$ \\
\hline pp2lq & 1.0000 & 1.0000 & $\lambda=5$ \\
\hline zn2ast & 4.2882 & 2.3397 & \\
\hline zn2br & 8.4320 & 3.2525 & \\
\hline zn2pi & 4.4683 & 3.4840 & \\
\hline zn2tak & 1.3598 & 1.0815 & \\
\hline zn3tak & 1.4491 & 1.2384 & \\
\hline \multicolumn{4}{|l}{} \\
\end{tabular}

Graphical representation of performance of individual control algorithms is expressed in Fig 10.

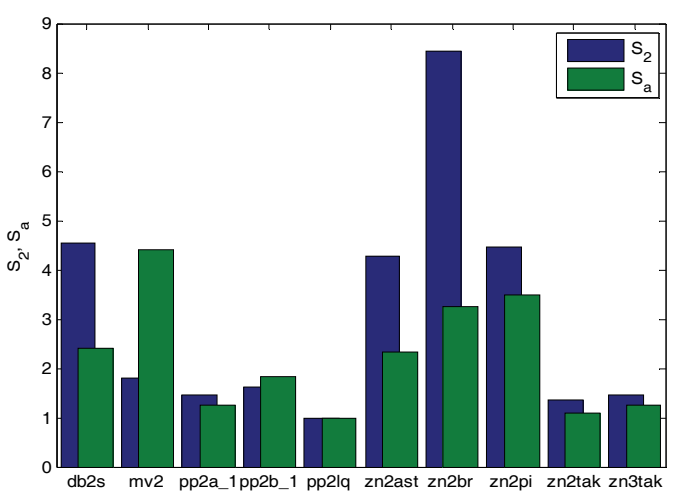

Figure 10: $S_{2}$ and $S_{a}$ Criteria for Different Algorithms

The $d b 2 s$ is a dead-beat controller. The $m v 2$ is a minimum variance controller, where $q$ is a penalisation coefficient. Controllers $p p 2 a \_1$ and $p p 2 b \_1$ are poleplacement controllers of the one degree of freedom structure and two degree of freedom structure respectively. Positions of the closed loop poles are computed to obtain a dynamic behaviour of the closed loop similar to the second order continuous system with characteristic polynomial

$$
d(s)=s^{2}+2 \xi \omega s+\omega^{2}
$$

The $p p 2 l q$ is the nominal LQ controller. The remaining controllers are based on Ziegler-Nichols tuning method.
The zn2ast is the Åström controller. The $z n 2 b r$ is a Ziegler-Nichols controller with backward rectangular method of integration. The $z n 2 p i$ is a Ziegler-Nichols PI controller. The zn2tak and the z3tak represent Takahashi's modification of the Ziegler-Nichols algorithm using second and third order model respectively.

The best performance was reached by the nominal $p p 2 l q$ controller but good results were achieved also by pp2a_1 pole-placement controller and by Takahashi's controllers. The control courses of the zn2tak controller are presented in the Fig. 11.
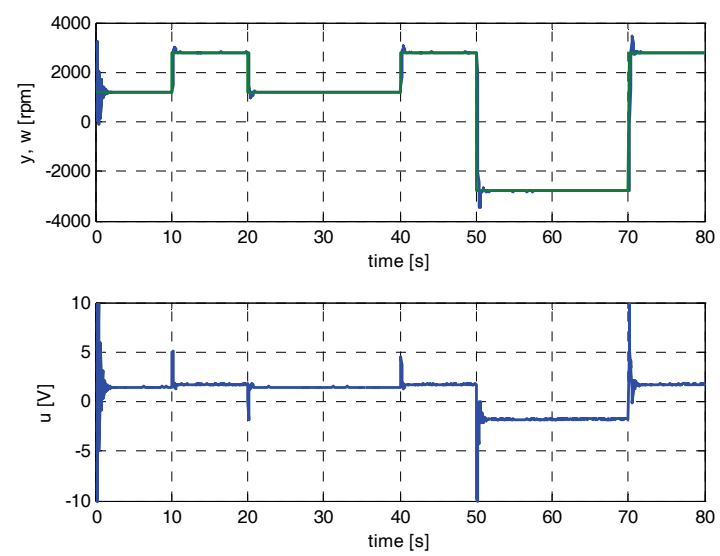

Figure 11: Control Courses of zn2tak controller

\section{Tuning of the pp2a_1 controller}

As can be seen from Table 5, a very good control performance was achieved by the $p p 2 a \_1$ controller. Different settings of parameters $\xi$ and $\omega$ were investigated and the results are summarized in Table 6. The sample time of $T_{0}=0.05 \mathrm{~s}$ and the adaptive directional forgetting identification method was used in all cases. The nominal $p p 2 l q$ controller is presented for the comparison purposes.

Table 6: Comparison of Different Settings of $p p 2 a \_1$

\begin{tabular}{|c|c|r|r|}
\hline$\xi$ & $\omega$ & $S_{2}[1]$ & $S_{a}[1]$ \\
\hline 0.5 & 2 & 2.5086 & 3.8248 \\
\hline 0.8 & 2 & 3.7334 & 2.3190 \\
\hline 1 & 1 & 15.2416 & 11.3481 \\
\hline 1 & 2 & 1.4491 & 1.2384 \\
\hline 1 & 5 & 6.2797 & 1.7654 \\
\hline 1 & 10 & 6.0036 & 1.7932 \\
\hline 1.5 & 2 & 3.3655 & 1.9910 \\
\hline 2 & 1 & 7.5373 & 8.4371 \\
\hline 2 & 2 & 3.5361 & 2.2033 \\
\hline \multicolumn{2}{|r|}{ nominal $p p 2 l q(\lambda=5)$} & 1.0000 & 1.0000 \\
\hline
\end{tabular}

The best performance was reached by $\xi=1$ and $\omega=2$ according to both $S_{2}$ and $S_{a}$ criteria. The control courses for the $\xi=1$ and $\omega=2$ setting are presented in Fig. 12. 

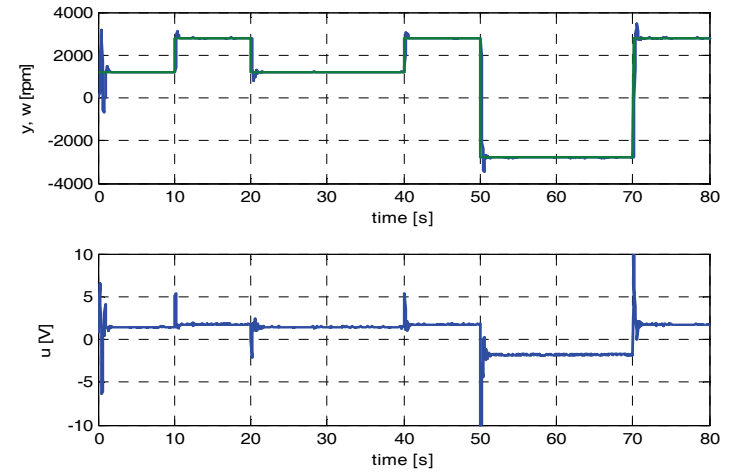

Figure 12: Control Courses of $p p 2 a \_l(\xi=1, \omega=2)$

\section{CONCLUSIONS}

Self-Tuning Controllers Simulink Library was introduced in the paper and controllers from this library were verified in the real-time conditions. Many controllers from the STCSL were tested by control of the Amira DR300 Servo System. This system in nonlinear and contains significant hysteresis.

The goal of the experiments was to find out optimal setting of individual controller algorithms. A set of tested parameters include sample time, control algorithm, forgetting of identification part and others. A convex behaviour was observed for all tested parameters which can be set continuously. Thus, an optimal value of the parameter can be found and change from this optimal setting leads to monotonous increase of a performance criteria. It should be noted that the problem of finding optimal setting of controller parameters is multidimensional, i.e. if other controller parameter changes, the optimal value of the investigated parameter can also change.

The best performance was achieved by LQ controller but satisfactory results were also produced by simpler Ziegler-Nichols based controllers.

\section{ACKNOWLEDGEMENTS}

This work was supported in part by the Ministry of Education of the Czech Republic under grants MSM 7088352101 and 1M0567 and partly by the Grant Agency of the Czech Republic under the grant No. 102/06/P286.

\section{References}

Bobál, V., J. Böhm, J. Fessl and J. Macháček. 2005. Practical Aspects of Self-tuning Controllers: Algorithms, Implementation and Applications. Springer-Verlag, London.

Bobál, V., J. Böhm, and R. Prokop. 1999. "Practical Aspects of Self-tuning Controllers". International Journal of Adaptive Control and Signal Processing, 13, 671-690.

Bobál, V. and P. Chalupa. 2002. "Self-tuning Controllers Simulink Library". http: //www.utb.cz/stctool/.

Bobál, V., P. Chalupa and P. Dostál. 2008. "Library for Design and Simulation Verification of Self-tuning
Controllers". In Proceedings of the $22^{\text {nd }}$ European Conference on Modelling and Simulation (Nicosia, Cyprus, June 3-6), 493-499.

Kučera, V. 1979. Discrete Linear Control: The Polynomial Equation Approach. John Wiley, Chichester.

Kučera, V. 1991. Analysis and Design of Discrete Linear Control Systems. Prentice Hall, London.

Kulhavý, R. 1987. "Restricted Exponential Forgetting in Real Time Identification". Automatica, 23, 586-600.

Ziegler, J. G. and N. B. Nichols. 1942. "Optimum Settings for Automatic Controllers". Trans. ASME, 64, 759 - 768.

\section{AUTHOR BIOGRAPHIES}

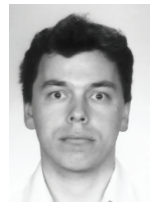

PETR CHALUPA was born in Zlin, Czech Republic in 1976. He graduated from Brno University of Technology in 1999. He obtained his Ph.D. in Technical Cybernetics at Tomas Bata University in Zlin in 2003. He works as a researcher at Centre of Applied Cybernetics at Tomas Bata University in Zlin. His research interests are adaptive and predictive control of real-time systems. You can contact him on email address chalupa@fai.utb.cz.

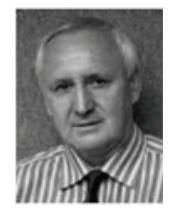

VLADIMÍR BOBÁL was born in Slavičín, Czech Republic in 1942. He graduated in 1966 from the Brno University of Technology. He received his Ph.D. degree in Technical Cybernetics at Institute of Technical Cybernetics, Slovak Academy of Sciences, Bratislava, Slovak Republic. He is now Professor in the Department of Process Control, Faculty of Applied Informatics of the Tomas Bata University in Zlín. His research interests are adaptive control systems, system identification and CAD for self-tuning controllers. You can contact him on email address bobal@fai.utb.cz.

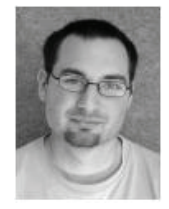

JAKUB NOVÁK was born in Zlin, Czech Republic and received the Ph.D. degree from the Tomas Bata University, Zlin, Czech Republic in 2007. He is now a researcher at the Center for Applied Cybernetics at Tomas Bata University. His research interests are multiple model strategies and predictive control. His e-mail address is: jnovak@fai.utb.cz

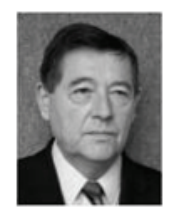

PETR DOSTÁL was born in Kněždub, Czech Republic in 1945. He studied at the Technical University of Pardubice, where he obtained his master degree in 1968 and $\mathrm{PhD}$. degree in Technical Cybernetics in 1979. In the year 2000 he became professor in Process Control. He is now head of the Department of Process Control, Faculty of Applied Informatics of the Tomas Bata University in Zlín. His research interests are modelling and simulation of continuous-time chemical processes, polynomial methods, optimal and adaptive control. You can contact him on email address dostalp@fai.utb.cz. 